\title{
Meisoindigo inhibits cellular proliferation via down-regulation of the PI3K/Akt pathway and induces cellular apoptosis in glioblastoma U87 cells
}

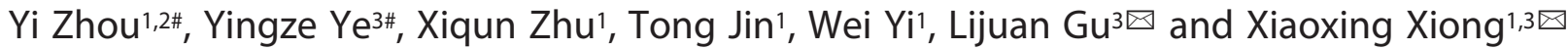 \\ 1Department of Neurosurgery, Renmin Hospital of Wuhan University, Wuhan, Hubei 430060, China; ${ }^{2}$ Department of Neurosurgery, Wuhan Asia \\ General Hospital, Wuhan, Hubei 430056, China; ${ }^{3}$ Central laboratory, Renmin Hospital of Wuhan University, Wuhan, Hubei 430060, China
}

\begin{abstract}
Objective: The current study was to explore whether meisoindigo was effective in suppressing proliferation and inducing apoptosis of human glioblastoma multiforme U87 cells and to explore its possible mechanisms. Method: Morphological changes were observed by light microscopy. Cell counting kit-8 (CCK-8) assay was performed to detect cellular proliferation. Apoptosis was monitored by flow cytometry. Akt, phospho-Akt, PI3K, p65, phospho-p65 and apoptosis-related proteins caspase- 3 and caspase- 9 were examined by Western blotting assays. Immunofluorescence was used to evaluate level of P65 expression in cells. Result: Meisoindigo inhibited the proliferation of U87 cells, and the inhibitory effect increased in a dose dependent manner. Moreover, meisoindigo exposure triggered an increase in the level of caspase- 3 and caspase-9, supporting its role in the activation of apoptosis. Furthermore, meisoindigo reduced the expression of PI3K, Akt, phospho-Akt, NF-KB, p65 and phospho-p65 in U87 cells, and displacement of p65 from the nucleus to the cytoplasm. Conclusion: Meisoindigo inhibits proliferation and induces apoptosis of U87 cells, probably through down-regulating the PI3K/Akt pathway and reducing nuclear translocation of NF-KB p65.
\end{abstract}

Keywords: meisoindigo, glioblastoma, cell proliferation, apoptosis, NF-KB

Received: 23 December, 2020; revised: 24 December, 2020 accepted: 12 January, 2021; available on-line: 23 May, 2021

घe-mail: xiaoxingxiong@whu.edu.cn (XX); gulijuan@whu.edu.cn (LG)

"Equally contributed to this paper.

Acknowledgments of Financial Support: This study was supported by the National Natural Science Foundation of China (No. 81771283 to LJ Gu and No. 81571147 to XX Xiong) and the Health and Family Planning Committee of Hubei Province (Grant of Traditional Chinese Medicine and Combination of Traditional Chinese and Western Medicine to $\mathrm{W} Y \mathrm{Yi}$.

Abbreviations: CCK-8, Cell counting kit-8; CML, chronic myeloid leukemia; GBM, glioblastoma multiforme; WHO, World Health Organization; TMZ, temozolomide

\section{INTRODUCTION}

There are multiple grades of gliomas - grade II, III and IV, with grade IV being the most malignant. Glioblastoma, sometimes referred to as glioblastoma multiforme (GBM), considered as grade IV tumor according to the World Health Organization (WHO) classification, is believed to be the most frequent and the highest malignant histological subtype glioma (Wirsching et al., 2016). It is well known for its "three-high and one- low" characteristics, which means a high incidence, a high mortality, and a high recurrence rate and a low cure rate, and it serious effects on human health (Nayak \& Reardon, 2017). Nowadays, the standard treatment for glioblastoma is surgery followed by chemotherapy based on temozolomide (TMZ) and radiotherapy, which leads to poor patient survival. However, the prognosis for this disease continues to be bleak, with a mean survival of only 15 months after diagnosis (Ohba \& Hirose, 2016). Autophagy, cellular senescence, programmed cell death and necrosis are key responses of a cell facing a stress. Autophagy is the key player in TMZ resistance in GBM. TMZ can induce apoptosis due to selective inhibition of autophagy, in which autophagic vehicles accumulate as their fusion with lysosomes is blocked (Pawlowska et al., 2018). GBM cells can be intrinsically resistant to apoptosis, which is one of the most important reasons for GBM treatment failure and high recurrence (Hottinger et al., 2014), and new therapeutic methods that can effectively control glioblastoma are urgently required.

Meisoindigo is one of the most common clinical antitumor drugs in clinical practice. It is a structural analogue of the natural ingredient of indirubin, widely used in the treatment of chronic myeloid leukemia (CML) in China since the 1980s (Xiao et al., 2000). Comparative clinical trials showed that meisoindigo was as effective for $\mathrm{CML}$ as hydroxyurea in hematological responses, median duration of chronic phase, median survival and blast crisis rate at 60 months from diagnosis. Furthermore, meisoindigo in combination with hydroxyurea prolonged the median duration of chronic phase and median survival while reduced incidence of blast crisis as compared to busulphan, meisoindigo or hydroxyurea alone, suggesting that meisoindigo has a synergistic effect with hydroxyurea (Huang et al., 2014). It inhibits DNA and RNA synthesis and enables assembly of microtubules in vitro. Nowadays, meisoindigo is mainly used in the treatment of leukemia (Huang et al., 2006). It induces not only differentiation of neutrophils via downregulating c-Myb probably, but also inhibits proliferation and apoptosis of AML cells (Lee et al., 2010) in vivo and in vitro. But now, several studies have shown that the growth of multiple tumor cells is also inhibited by meisoindigo, such as colorectal cancer HT-29 cells and JAK2/V617F heterozygous mutation cell line-SET2 cell line (Mingxin et al., 2008). Meisoindigo can also affect the Wnt signal pathway through inhibiting the GSK-3beta expression and down-regulating the beta-catenin and c-MYC protein expression, which play an important role in the treatment for chronic myeloid leukemia (Zuo et al., 2010). Moreover, its anti-tumor effect is much more 
obvious and adverse reaction is much milder than natural indirubin (Xiao et al., 2002). Unfortunately, its antiglioma effect has not been reported so far.

The PI3K/Akt signaling pathway, one of the crucial cellular signal pathways, plays a pivotal role in inhibiting apoptosis as well as promoting cellular proliferation (Shi et al., 2018; Zhang et al., 2018). It is extremely closely related to the occurrence and development of various kinds of human cancers (Robbins \& Hague, 2015; Spangle et al., 2017). PI3K/Akt is characterized by persistent phosphorylation in a large number of malignant tumors including glioblastoma (Wang et al., 2017). The over-activation promotes downstream gene expression, resulting in uncontrolled cell growth, inhibition of tumor cell apoptosis, promotion of tumor cell proliferation, facilitating tumor immune escape, and ultimately leading to the occurrence and development of malignant tumors ( $\mathrm{Li}$ et al., 2016). Therefore, the PI3K/Akt pathway is an attractive potential target for glioblastoma treatment.

Studies have shown that a series of drugs could inhibit the growth of tumor cells as well as induce the expression of apoptosis-related proteins by inhibiting some active molecules related to the PI3K/Akt pathway (Huang et al., 2017; Zhang et al., 2018). We consequently studied the effect of meisoindigo on human astrocytoma cell line U87 in vitro and explored its possible mechanism so as to provide a pre-clinical basis for extending the application of meisoindigo.

\section{MATERIALS AND METHODS}

\section{Cell line and agents}

Human glioblastoma cell line U87-CL-0238, Procell, Wuhan, China, was cultured in RPMI-1640 (G4530, Servicebio, Wuhan, China) with 10\% fetal bovine serum (FBS, 11011-8611, Every Green, Hangzhou, China) at $37^{\circ} \mathrm{C}$ at an atmosphere containing $5 \%$ of $\mathrm{CO}_{2}$ with saturated humidity, and logarithmically growing cells were collected for further experiments.

Anti-caspase-3 (\# 9662S), anti-Akt (\# 9272S), antiphospho-Akt (\# 4060S), anti-PI3K (\# 4228S), anti-p65 (\# 8242S) and anti-phospho-p65 (\# 3031S) antibodies were purchased from Cell Signaling Technology (CST, Boston, United States). Anti-caspase-9 antibody (ab32539) was obtained from Abcam (Cambridge, United Kingdom). Annexin-V kit was purchased from BD Biosciences (556422, Franklin Lakes, United States). Alexa 594-conjugated antibody and Alexa 488-conjugated antibody were purchased from Millipore (Billerica, MA, United States). IRDye-labeled secondary antibody was purchased from Li-Cor Bioscience (United States) The BCA kit (P0012S) and CCK-8 kit (C0038) were purchased from Beyotime (Shanghai, China).

\section{Cell viability determination}

After rinsing with PBS buffer solution (G4202, Servicebio, Wuhan, China), U87 cells were seeded in 96well plates and cultured for $24 \mathrm{~h}$. Dimethyl sulfoxide was used as an organic solvent to dissolve meisoindigo and then PBS was used to regulate the concentration. Then, U87 cells were exposed to serial concentrations of meisoindigo (\#1011055-201001, National Institutes for Food and Drug Control, Beijing, China) for $24 \mathrm{~h}$. Finally, CCK- 8 solution was added into each well and incubated at $37^{\circ} \mathrm{C}$ for additional $4 \mathrm{~h}$ before spectrophotometry measurement at $590 \mathrm{~nm}$. The experiment was repeated three times independently. The cells were stained with trypan blue and counted with a cell count plate. The cell growth inhibition rate was calculated by the formula: the cell growth inhibition rate $(\%)=(1-$ the experimental group/the control group) $\times 100 \%$, and IC50 was obtained by statistical calculation.

\section{Morphological observation}

The cells were treated with serial concentrations of meisoindigo for $24 \mathrm{~h}$. The morphological characteristics of the cells were observed under the automatic fluorescence microscope (BX63, Olympus Optical Ltd, Tokyo, Japan).

\section{Determination of apoptosis rate}

Logarithmically growing cells were collected and treated with serial concentrations of meisoindigo for $24 \mathrm{~h}$. After wash with cold PBS, labeled and assayed with reference to antibody instructions, the cells were added to FITC labeled RNA enzyme Annexin V and propidium iodide pyridine (PI), respectively. The cells were incubated for $1 \mathrm{~h}$ in the dark and then detected by flow cytometry (BD, Biosciences, Franklin Lakes, NJ, USA). At the same time, the apoptosis rate was calculated. This experiment was repeated three times and the average was taken.

\section{Western blotting assays}

U87 cells from different groups were collected and lysed with RIPA buffer containing protease inhibitors and phosphates inhibitors (G2002, Servicebio, Wuhan, China). The supernatant was collected after centrifugation at $14000 \mathrm{rpm}$ for $20 \mathrm{~min}$ at $4^{\circ} \mathrm{C}$, and the protein concentration was quantified with the use of BCA kit. The total protein was separated by electrophoresis in $10 \%$ polyacrylamide gel loaded at $30 \mu \mathrm{g} /$ well. Proteins were transferred to PVDF membrane (Millipore, Billerica, MA, United States) after electrophoresis. The membrane was firstly incubated in Tris-buffered saline containing $5 \%$ fat-free milk and $0.1 \%$ Tween 20 for blocking for 1 $\mathrm{h}$ at room temperature, and then incubated with primary antibodies at $4^{\circ} \mathrm{C}$ overnight, followed by incubation with horseradish peroxidase-conjugated secondary antibodies (Santa Cruz Biotechnology). Then, the membrane was scanned with Bio-Rad ChemiDoc Imaging System (BIO-RAD Life Science Research, Hercules, CA, USA) and the gray scale values were measured with the use of Quantity One v4.6.2 software (Bio-Rad Laboratories, Hercules, CA, United States). The relative level of protein expression is: the gray value of the different sample protein bands/gray value of the corresponding sample actin and GADPH protein band.

\section{Immunofluorescence staining}

Cells cultured on $22 \times 22 \mathrm{~mm}$ (No. 1.5) coverslips were treated with 10, 20 and $30 \mu \mathrm{mol} / \mathrm{L}$ of meisoindigo, left to rest for $24 \mathrm{~h}$ before fixation with $100 \%$ methanol for 3 minutes at $-20^{\circ} \mathrm{C}$. Then, the coverslips were washed three times with PBS $+1 \%$ normal goat serum (NGS, 5452S, CST, Boston, United States), incubated in a humid chamber with the primary p65 antibody for $1 \mathrm{~h}$ at a room temperature, washed three times with PBS plus $1 \%$ NGS, each for 10 minutes, and incubated for $1 \mathrm{~h}$ at a room temperature in a humid chamber with a fluorescently labeled secondary antibody diluted to $4 \mu \mathrm{g} / \mathrm{mL}$. Washed four times with PBS, 10 minutes each time. The coverslip was sealed on the carrier sheet with a lidding 
A

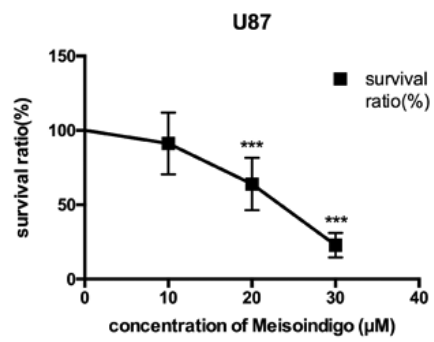

C

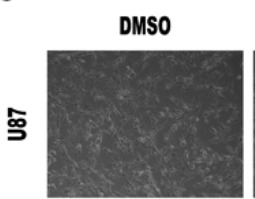

B

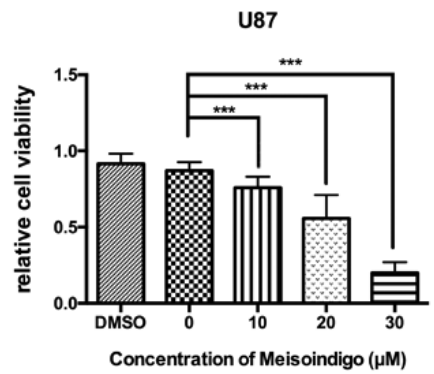

Figure 1. Inhibitory effects of meisoindigo on the proliferation of U87 cells.

A) Meisoindigo inhibits the proliferation of U87 cells in a dose-dependent manner. The IC50 value is 20umol/L B) Relative cell viability of cells in the treatment group was significantly decreased when comparing with the control group. C) The morphological changes of U87 cells are observed under microscope. As the concentration of meisoindigo increases, the number of total and adherent cells decreases. Mean \pm S.D., $\mathrm{n}=5$. ${ }^{* * *} P<0.001$ vs $0 \mu \mathrm{mol} / \mathrm{L}$.

agent and clean nail polish was used to prevent the coverslip from sliding. It was then immediately observed under an automatic fluorescence microscope (BX63, Olympus Light Ltd, Tokyo, Japan).

\section{Statistical analysis}

SPSS 18.0 (SPSS, Chicago, IL, USA) was used for statistical analysis. All data were presented as mean \pm S.D. One-way ANOVA was used to compare numerical data in more than 2 groups. $P<0.05$ was considered statistically significant.

\section{RESULTS}

\section{Effect of Meisoindigo on the proliferation of U87 cells}

U87 cells were treated with different concentrations $(10,20$ and $30 \mu \mathrm{mol} / \mathrm{L})$ of meisoindigo for $24 \mathrm{~h}$, the proliferation of U87 cells was inhibited by meisoindigo in a dose-dependent manner. The IC50 was $20 \mu \mathrm{mol} / \mathrm{L}$ $(P<0.001)$ (Fig. 1A, B).

\section{Effect of meisoindigo on the morphology of U87 cells}

U87 cells were subjected to the same treatment as in 2.1. Then, it was observed that as the drug concentration gradient accelerates, the number of total cells decreased $(P<0.001)$ (Fig. 1C).

\section{Effect of meisoindigo on the apoptosis rate and expression of apoptosis-related proteins in U87 cells}

U87 cells were treated with the same gradients of meisoindigo as in Materials and Methods. The cellular apoptosis rate and the expression of apoptosis-related proteins were measured with the use of flow cytometry and Western blotting assays, respectively. The level of cellular apoptosis induced by meisoindigo was dosedependent, and the difference was statistically significant

\section{A}

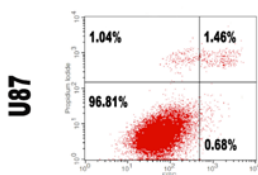

DMSO

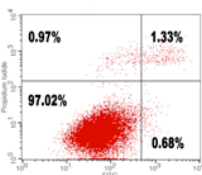

Opmol/L

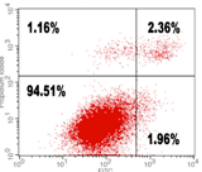

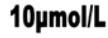

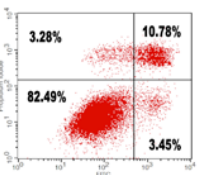

$20 \mu \mathrm{mol} / \mathrm{L}$

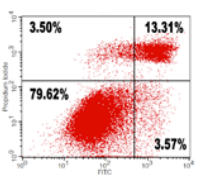

30pmol/L
B

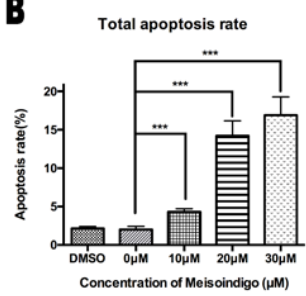

C

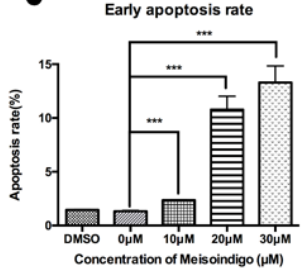

D

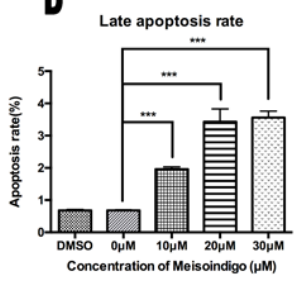

Figure 2. Effect of meisoindigo on U87 cells in inducing apoptosis.

A) Results from flow cytometry. The cells in the lower-left quadrant are normal cells, the cells in upper-right and lower-right quadrant are late and early apoptotic cells, respectively. B-D) Cellular apoptosis induced by meisoindigo is dose-dependent, with statistically significant difference when compared with the control group. Mean \pm S.D., $n=5$. ${ }^{* * *} P<0.001 \mathrm{vs} .0 \mu \mathrm{mol} / \mathrm{L}$. 

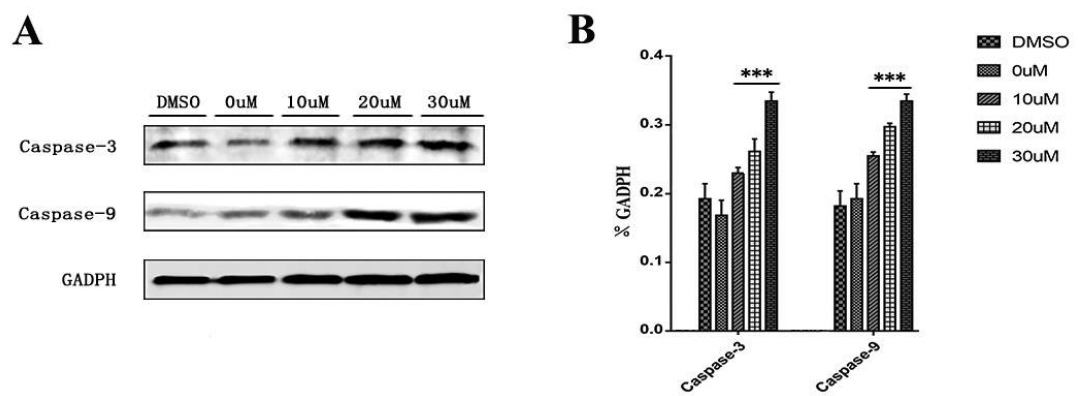

Figure 3. Effect of meisoindigo on the expression of apoptosis-related proteins, based on Western blot analysis.

Compared with the control group, the expression level of caspase- 3 and caspase- 9 is increased by meisoindigo treatment in a dosedependent manner, and the statistical difference is significant. Mean \pm S.D., $\mathrm{n}=5$. ${ }^{* * *} P<0.001 \mathrm{vs} .0 \mu \mathrm{mol} / \mathrm{L}$

compared with the control group $(P<0.001)$ (Fig. 2). In addition, contrasting to the control group, caspase- 3 and caspase-9, as well-known and reliable apoptosis-related proteins, were also significantly increased after meisoindigo treatment in a dose-dependent manner $(P<0.001)$ (Fig. 3).

\section{Molecular mechanism of meisoindigo on inhibiting proliferation and inducing apoptosis}

Meisoindigo inhibited the phosphorylation of Akt in U87 cells. Meisoindigo at $20 \mu \mathrm{mol} / \mathrm{L}$ significantly inhibited Akt activation in U87 cells. In the meantime, the level of PI3K, the upstream molecule of Akt, was detected. These results indicated that the expression of these two groups of protein was also inhibited and this effect was statistically significant $(P<0.001)$ (Fig. 4). The level of nuclear protein p 65 and its phosphorylation was also found to be significantly different $(P<0.001)$ (Fig. 4).

\section{Effect of meisoindigo on P65 protein in U87 cells}

$\mathrm{NF}-x \mathrm{~B}$ is a transcriptional factor regulating a wide array of genes mediating numerous cellular processes such as proliferation, differentiation, motility and survival (Hayden \& Ghosh, 2012; Perkins, 2007). In addition, aberrant activation of $\mathrm{NF}-x \mathrm{~B}$ is a frequent event in numerous cancers, including glioblastoma (Soubannier \& Stifani, 2017). Therefore, we examined the effect of meisoindigo on the activation of NF- $x \mathrm{~B}$ p 65 with immunofluorescence analysis. The result showed that administration of meisoindigo by different concentrations of $10,20,30 \mu \mathrm{mol} / \mathrm{L}$ markedly downregulated the protein expressions of $\mathrm{NF}-x \mathrm{~B}$ p65 in dose dependent manners $(P<0.05)$. (Fig. 5).

\section{DISCUSSION}

Nowadays, the main treatment regimen of human glioblastoma includes surgery, chemotherapy, radiotherapy, endocrine therapy and targeted therapy (Ohba \& Hirose, 2016; Wirsching et al., 2016). However, it is characterized by uncontrolled cell proliferation, highly diffusive infiltration, resistance to apoptosis, robust angiogenesis, and DNA repair mechanisms contributing to drug resistance. When the extent of DNA damage excesses DNA repair potential, such as in the case of many anticancer drugs,

\section{B}

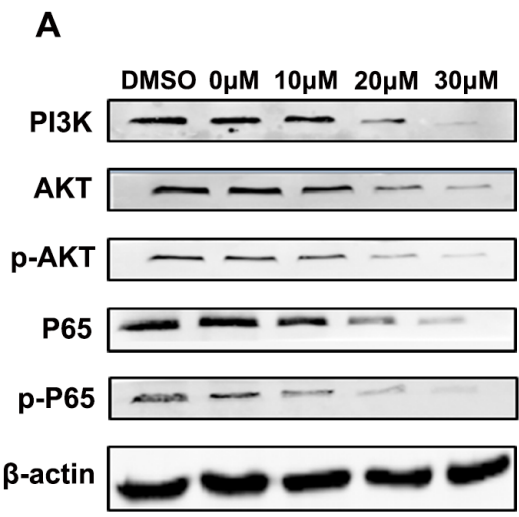

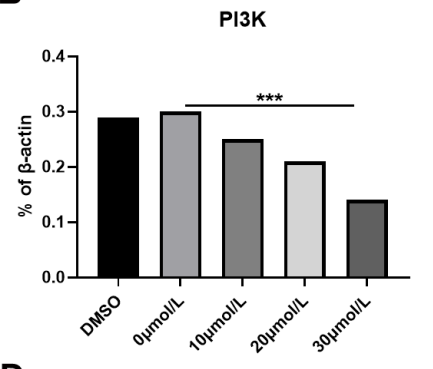

D

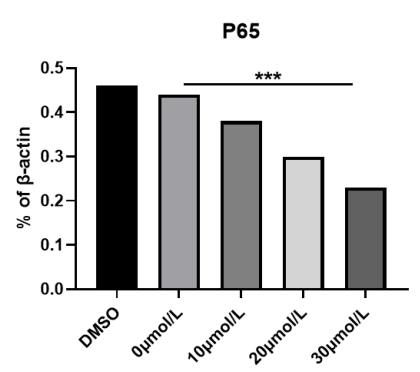

C

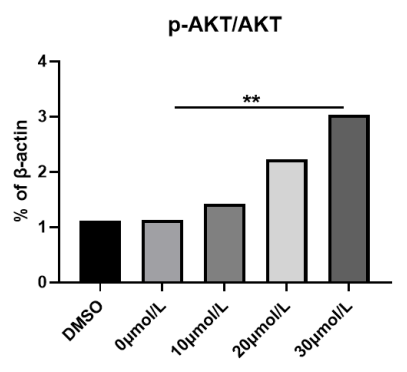

E

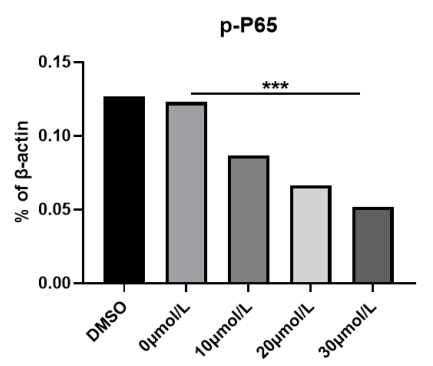

Figure 4. Molecular mechanism whereby meisoindigo inhibits proliferation and induces apoptosis.

Meisoindigo inhibits the phosphorylation of Akt in U87 cells. Meisoindigo at $20 \mu \mathrm{mol} / \mathrm{L}$ can significantly inhibit Akt activation in U87 cells. In the meantime, the level of PI3K, the upstream molecule of Akt, is detected. These results indicate that the expression of these two groups of protein is also inhibited and this effect is statistically significant. Mean \pm S.D., $n=5 .{ }^{* *} P<0.01 \mathrm{vs} .0 \mu \mathrm{mol} / \mathrm{L},{ }^{* * *} P<0.001 \mathrm{vs}$. $0 \mu \mathrm{mol} / \mathrm{L}$ 
A
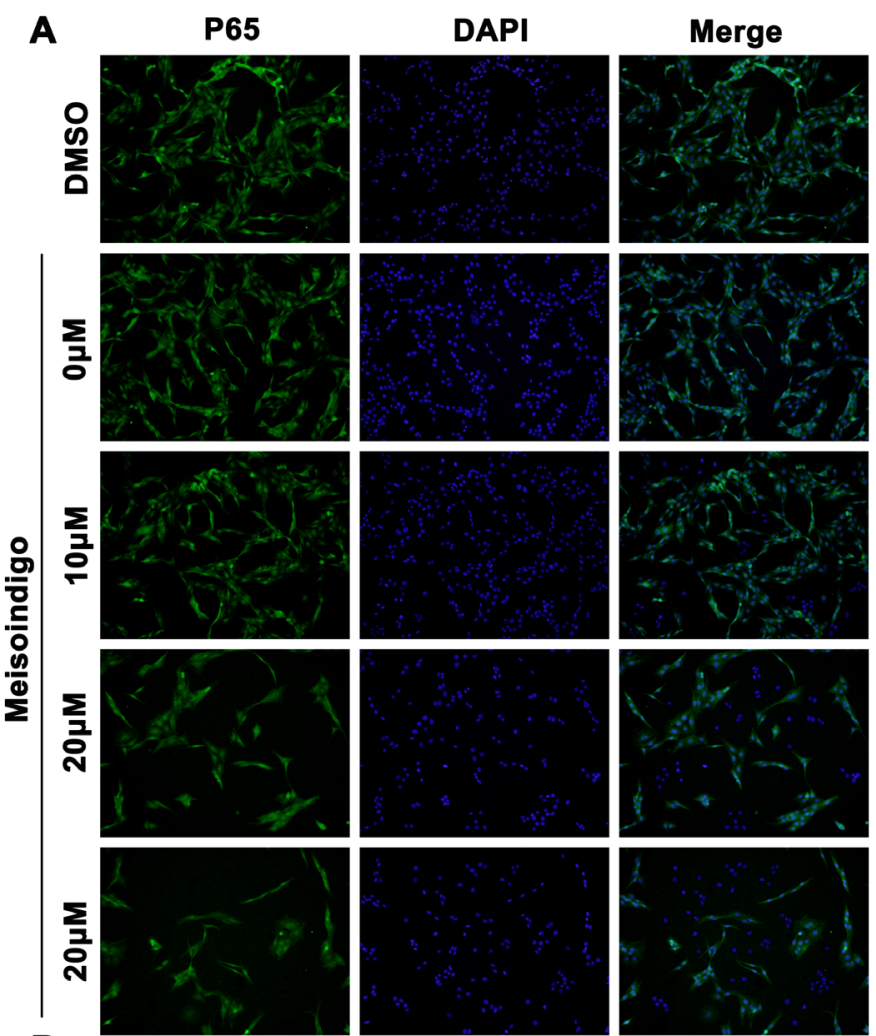

B

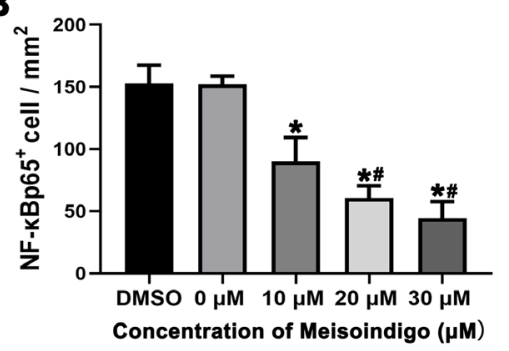

Figure 5. Effect of meisoindigo on P65 protein in U87 cells.

The level of nuclear protein P65 and its nuclear translocation is significantly different. A) Representative imaging of for nuclear protein P65, counterstained with DAPI after different dose (0, 10, 20 and $30 \mu \mathrm{M})$ of Meisoindigo treatment. B) Quantification of P65-positive cells in U87 cell after different dose of Meisoindigo treatment. Mean \pm S.D., $\mathrm{n}=5$. Scale bar $=20 \mathrm{~mm}$. ${ }^{*} P<0.05$ vs. DMSO; ${ }^{2} P<0.05$ vs.10 $\mu \mathrm{M}$ Meisoindigo.

a cell can be given, or gives itself, an additional time for that damage repair at the $\mathrm{G} 1 / \mathrm{S}$ or $\mathrm{G} 2 / \mathrm{M}$ checkpoint of the cell cycle or be directed on a programmed death pathway, usually apoptosis (Lin et al., 2017). However, cancer cells can avoid this scenario and survive treatment with anticancer drugs due to deregulated DNA damage response and apoptosis resistance. Autophagy, a process of removal of damaged or no longer needed cellular products, has two faces in response to TMZ and other anticancer drugs, pro-survival and pro-death, resulting in the opposite effects, TMZ resistance and TMZ enhanced sensitivity, respectively (Klionsky \& Schulman, 2014). Autophagy can protect against apoptotic death, but, when too extensive, can lead to self-destruction of a cancer cell. On the other hand, inhibition of autophagy can result in apoptosis activation and cell death, but when a cancer cell is intrinsically resistant to apoptosis, accumulation of toxic waste not cleared by autophagy takes place. Therefore, it is very important to seek more effective drugs against human glioblastoma.

Meisoindigo, an indirubin compound, is an anti-cancer drug developed in China. Clinical and laboratory studies have shown that meisoindigo is effective as an anti-leukemia drug (Huang et al., 2014; Xiao et al., 2002). Both animal models and clinical trials showed that meisoindigo displayed higher antitumor ability than indirubin (Ji \& Zhang, 1985). In maintenance treatment, meisoindigo produced less transformation and longer survival in CML patients compared to busulfan, another classic drug for CML treatment. However, further studies of meisoindigo as a treatment of CML became tarnished as imatinib became a better choice for CML treatment. Experimental results on the mouse leukemia L1210 cell cycle showed that meisoindigo induced accumulation of $\mathrm{S}$ phase cells. The movement of cells in G2+M phase to G1 phase may also be blocked to some extent (Ji et al., 1991). Another study suggested that the induction of cancer cell differentiation associated with decreased c-myb oncogene expression might also account for the anticancer action and low toxicity of meisoindigo (Liu et al., 1996). In addition, Xiao and his team found that meisoindigo could inhibit an angiogenetic process through reducing the secretion of VEGF in leukemic cells and also through inhibiting the proliferation, adhesion and differentiation of 
endothelial cells, causing the interruption of a reciprocal stimulatory loop between leukemic and endothelial cells (Xiao et al., 2006). Moreover, one research showed that the antileukemic effects of meisoindigo were investigated in four human leukemic cells (NB4, NB4.007/6, HL60 and U937) including both retinoic acid sensitive and retinoic acid resistant cells, and meisoindigo could effectively inhibit the growth and/or proliferation of these four cell types (Huang et al., 2014). In view of meisoindigo as a lead compound possessing anti-leukemia effect with clinical safety, exploring its novel biological activities is extremely urgent for drug development in other diseases besides CML (Zheng et al., 2017). Moreover, its outstanding anti-cancer effect and mild adverse reactions suggest its potential application for the treatment of solid tumor, for example human glioblastoma (Lee et al., 2010). The present study aimed to fill this gap in knowledge by exploring the in vitro inhibitory effects of meisoindigo in human glioblastoma cell lines and the underlying signaling mechanism.

We studied the effect of meisoindigo on human glioblastoma cell line U87. The results showed that meisoindigo can significantly inhibit the proliferation of U87 cells in a dose dependent manner with an IC50 of 20 $\mu \mathrm{mol} / \mathrm{L}$, which was observed with the use of light microscopy after U87 cells were treated with meisoindigo for $24 \mathrm{~h}$. The apoptosis rate was positively correlated with the concentration of meisoindigo. Flow cytometric results indicated that meisoindigo could inhibit the proliferation of U87 cells by inducing apoptosis as reported before (Mingxin et al., 2008). Furthermore, Western blotting assays showed that the treatment with meisoindigo significantly increased the expression of caspase- 3 and -9 in human glioblastoma cell line U87 in a dose-dependent manner.

Studies have shown that meisoindigo can regulate the conduction of signaling pathways (Zhang et al., 2013; Zuo et al., 2010), which are closely related to the tumor genesis. The phosphatidylinositol three kinase/protein kinase (PI3K/AKT) signaling pathway is an important regulatory pathway in cell cycle progression (Hawkins \& Stephens, 2015), which is closely related to the growth, differentiation and apoptosis of cells (Robbins \& Hague, 2015; Spangle et al., 2017). In this study, we found that the expression of Akt, p-Akt, PI3K, p65 and phosphop65 nuclear protein were down-regulated by meisoindigo, and intracellular content of P65 was also observed with immunofluorescence microscopy, suggesting that the apoptosis of U87 cells induced by meisoindigo may be regulated by the PI3K/AKT pathway and may be associated with nuclear translocation. Furthermore, a study showed that meisoindigo has neuro-protective effect on ischemic brain, the protection attributes to its ability of relieving the brain inflammation by inhibiting the activation of the NLRP3 inflammasome and preventing the microglial/macrophage switch from the pro-inflammatory M1 phenotype to the protective M2 phenotype, which may obtain through the inhibition of TLR4/NF$x \mathrm{~B}$ signaling pathway in neurons and microglia (Ye et al., 2019). Due to the synergistic effect of meisoindigo with hydroxyurea in CML, we assumed that meisoindigo might have the same effect with other chemotherapeutics in GBM.

This study demonstrated that meisoindigo could induce apoptosis of U87 cells and inhibit their proliferation, and the preliminary molecular mechanism underlying apoptosis. Our studies suggest that meisoindigo has a significant anti-proliferation effect and induces apoptosis on human glioblastoma cell line U87, which may guide a wider application of meisoindigo in clinical practice. However, further experiments on other glioblastoma cell lines or primary cells collected from human glioblastoma samples are needed to confirm the effect of meisoindigo on glioblastoma, and further studies are necessary to elucidate the mechanisms and complex molecules involved in this process. To explore late-stage apoptosis and other such delayed effects, it is needed to extend culture time of U87, and in vivo and in vitro validation experiments should be considered as well.

\section{Conflicts of interest}

The authors declare that there is no conflict of interests regarding the publication of this paper.

\section{Ethics approval and consent to participate}

This study does not contain any studies with human participants or animals performed by any of the authors. All procedures were conducted according to the guidelines of the bioinformatic criteria of China Wuhan University. The study was approved by the ethics Committee of Renmin Hospital of Wuhan University.

\section{Consent for publication}

The datasets used and/or analyzed during the current study are available from the corresponding author on reasonable request.

\section{Availability of data and materials}

Not applicable.

\section{REFERENCES}

Hawkins PT, Stephens LR (2015) PI3K signalling in inflammation. Biochim Biophys Acta 1851: 882-897. https://doi.org/10.1016/j.bbalip.2014.12.006

Hayden MS, Ghosh S (2012) NF-kappaB, the first quarter-century: remarkable progress and outstanding questions. Genes Dev 26: 203234. https://doi.org/10.1101/gad.183434.111

Hottinger AF, Stupp R, Homicsko K (2014) Standards of care and novel approaches in the management of glioblastoma multiforme. Chin J Cancer 33: 32-39. https://doi.org/10.5732/cjc.013.10207

Huang H, Li LJ, Zhang HB, Wei AY (2017) Papaverine selectively inhibits human prostate cancer cell (PC-3) growth by inducing mitochondrial mediated apoptosis, cell cycle arrest and downregulation of NF-xB/PI3K/Akt signalling pathway. J Buon 22: 112-118. PMID: 28365943

Huang M, Lin HS, Lee YS, Ho PC (2014) Evaluation of meisoindigo, an indirubin derivative: in vitro antileukemic activity and in vivo pharmacokinetics. Int J Oncol 45: 1724-1734. https://doi. org/10.3892/ijo.2014.2548

Ji XJ, Liu XM, Li K, Chen RH, Wang LG (1991) Pharmacological studies of meisoindigo: absorption and mechanism of action. Biomed Environ Sci 4: 332-337. PMID: 1764225

Ji XJ, Zhang FR (1985) Studies on antineoplastic action of indirubin derivatives and analogs and their structure-activity relationships. Yao Xue Xue Bao 20: 137-139. PMID: 4036618 (in Chinese)

Klionsky DJ, Schulman BA (2014) Dynamic regulation of macroautophagy by distinctive ubiquitin-like proteins. Nat Struct Mol Biol 21: 336. https://doi.org/010.1038/nsmb.2787

Lee CC, Lin CP, Lee YL, Wang GC, Cheng YC, Liu HE (2010) Meisoindigo is a promising agent with in vitro and in vivo activity against human acute myeloid leukemia. Leuk Lymphoma 51: 897-905. https://doi.org/10.3109/10428191003672115

Li X, Wu C, Chen N, Gu H, Yen A, Cao L, Wang E, Wang L (2016) $\mathrm{PI} 3 \mathrm{~K} / \mathrm{Akt} / \mathrm{mTOR}$ signaling pathway and targeted therapy for glioblastoma. Oncotarget 7: 33440-33450. https://doi.org/10.18632\%oncotarget.7961

Lin AB, McNeely SC, Beckmann RP (2017) Achieving precision death with cell-cycle inhibitors that target DNA replication and repair. Clin Cancer Res 23: 3232-3240. https://doi.org/10.1158/1078-0432. CCR-16-0083

Liu XM, Wang LG, Li HY, Ji XJ (1996) Induction of differentiation and down-regulation of c-myb gene expression in ML-1 human myeloblastic leukemia cells by the clinically effective anti-leukemia 
agent meisoindigo. Biochem Pharmacol 51: 1545-1551. https://doi. org/10.1016/0006-2952 (96)00098-6

Mingxin Z, Yan L, Hongbo W, Jianhua Z, Hongyan L, He L, Hongqi $\mathrm{X}$, Sen Z, Xiaoguang C (2008) The antitumor activity of meisoindigo against human colorectal cancer HT-29 cells in vitro and in vivo. J Chemother 20: 728-733. https://doi.org/10.1179/joc.2008.20.6.728

Nayak L, Reardon DA (2017) High-grade gliomas. Continuum (Minneap Minn) 23 (6, Neuro-oncology): 1548-1563. https://doi.org/10.1212/ con.0000000000000554

Ohba S, Hirose Y (2016) Current and future drug treatments for glioblastomas. Curr Med Chem 23: 4309-4316. https://doi.org/10.2174/ 0929867323666161014132907

Pawlowska E, Szczepanska J, Szatkowska M, Blasiak J (2018) An interplay between senescence, apoptosis and autophagy in glioblastoma multiforme-role in pathogenesis and therapeutic perspective. Int J Mol Sci 19: 889. https://doi.org/10.3390/ijms19030889

Perkins ND (2007) Integrating cell-signalling pathways with NF-kap$\mathrm{paB}$ and IKK function. Nat Rev Mol Cell Biol 8: 49-62. https://doi. org $/ 10.1038 / \mathrm{nrm} 2083$

Robbins HL, Hague A (2015) The PI3K/Akt pathway in tumors of endocrine tissues. Front Endocrinol (Lausanne) 6: 188. https://doi. $\mathrm{org} / 10.3389$ /fendo.2015.00188

Shi X, Yang L, Xie J, Zhao Y, Cong J, Li Z, Li H, Cheng X, Fan J (2018) UNBS5162 inhibits proliferation of human melanoma cells by inducing apoptosis via the PI3K/Akt pathway. Mol Med Rep 18: 3382-3388. https://doi.org/10.3892/mmr.2018.9321

Soubannier V, Stifani S (2017) NF-kappaB signalling in glioblastoma. Biomedicines 5: 29. https://doi.org/10.3390/biomedicines5020029

Spangle JM, Roberts TM, Zhao JJ (2017) The emerging role of PI3K/ AKT-mediated epigenetic regulation in cancer. Biochim Biophys Acta 1868: 123-131. https://doi.org/10.1016/j.bbcan.2017.03.002

Wang Y, Kuramitsu Y, Baron B, Kitagawa T, Tokuda K, Akada J, Maehara SI, Maehara Y, Nakamura K (2017) PI3K inhibitor LY294002, as opposed to wortmannin, enhances AKT phosphorylation in gemcitabine-resistant pancreatic cancer cells. Int J Oncol $\mathbf{5 0}$ 606-612. https://doi.org/10.3892/ijo.2016.3804

Wirsching HG, Galanis E, Weller M (2016) Glioblastoma. Handb Clin Neurol 134: 381-397. https://doi.org/10.1016/B978-0-12-802997$8.00023-2$
Xiao Z, Hao Y, Liu B, Qian (2002) Indirubin and meisoindigo in the treatment of chronic myelogenous leukemia in China. Leuk Lymphoma 43: 1763-1768. https://doi.org/10.1080/1042819021000006295

Xiao Z, Qian L, Liu B, Hao Y (2000) Meisoindigo for the treatment of chronic myelogenous leukaemia. Br I Haematol 111: 711-712. https://doi.org/10.1046/j.1365-2141.2000.02357.x

Xiao Z, Wang Y, Lu L, Li Z, Peng Z, Han Z, Hao Y (2006) Antiangiogenesis effects of meisoindigo on chronic myelogenous leukemia in vitro. Leukemia Res 30: 54-59. https://doi.org/10.1016/j. leukres.2005.05.012

Ye Y, Jin T, Zhang X, Zeng Z, Ye B, Wang J, Zhong Y, Xiong X, Gu L (2019) Meisoindigo protects against focal cerebral ischemiareperfusion injury by inhibiting NLRP3 inflammasome activation and regulating microglia/macrophage polarization via TLR4/ NF- $x$ B signaling pathway. Front Cell Neurosci 13: 553. https://doi. org/10.3389/fncel.2019.00553

Zhang B, Liu Y, Li Y, Zhe X, Zhang S, Zhang L (2018) Neuroglobin promotes the proliferation and suppresses the apoptosis of glioma cells by activating the PI3K/AKT pathway. Mol Med Rep 17: 27572763. https://doi.org/10.3892/mmr.2017.8132

Zhang HJ, Zhang Y, Jin J, Zhou WQ, Chen XG (2013) Mechanism about therapeutic effect of meisoindigo on psoriasis via down-regulation of the TLR4-TAK-NF-kappaB pathways. Yao Xue Xue Bao 48: 503-507. PMID: 23833936 (in Chinese)

Zhang X, Lan D, Ning S, Ruan L (2018) Anticancer action of lactucopicrin in SKMEL-5 human skin cancer cells is mediated via apoptosis induction, G2/M cell cycle arrest and downregulation of $\mathrm{m}=\mathrm{TOR} / \mathrm{PI} 3 \mathrm{~K} / \mathrm{AKT}$ signalling pathway. J Buon 23: 224-228. PMID: 29552788

Zheng XT, Wu ZH, Wei Y, Dai JJ, Yu GF, Yuan F, Ye LC (2017) Induction of autophagy by salidroside through the AMPK-mTOR pathway protects vascular endothelial cells from oxidative stressinduced apoptosis. Mol Cell Biochem 425: 125-138. https://doi. org/10.1007/s11010-016-2868-x

Zuo MX, Li Y, Zhou JH, Wang HB, Chen XG (2010) Effect of Meisoindigo on Wnt signal pathway in K562 and HL-60 cells. Zhongguo Shi Yan Xue Ye Xue Za Zhi 18: 579-582. PMID: 20561405 (in Chinese) 